This is an Open Access article, distributed under the terms of the Creative Commons Attribution licence (http://creativecommons.org/licenses/by/4.0/), which permits unrestricted re-use, distribution, and reproduction in any medium, provided the original work is properly cited.

doi:10.1017/jfm.2020.181

\title{
Minuet motion of a pair of capsules interacting in simple shear flow
}

\author{
X.-Q. Hu ${ }^{1,2}$, X.-C. Lei ${ }^{2}$, A.-V. Salsac ${ }^{1} \dagger$ and D. Barthès-Biesel ${ }^{1}$ \\ ${ }^{1}$ Biomechanics and Bioengineering Laboratory (UMR CNRS 7338), Université de Technologie de \\ Compiègne, Alliance Sorbonne Université, CS 60319, 60203 Compiègne, France \\ ${ }^{2}$ State Key Laboratory of Advanced Design and Manufacturing for Vehicle Body, College of \\ Mechanical and Vehicle Engineering, Hunan University, 410082 Changsha, China
}

(Received 29 September 2019; revised 22 February 2020; accepted 28 February 2020)

We study the three-dimensional hydrodynamic interaction of a pair of identical, initially spherical capsules freely suspended in a simple shear flow under Stokes flow conditions. The capsules are filled with a Newtonian liquid (same density and viscosity as the suspending fluid). Their membranes satisfy the neo-Hookean constitutive law. We consider the rarely studied case where the capsule centres are initially located in (or near) the plane defined by the flow direction and the vorticity vector, i.e. in two different shear planes. The motion and deformation of the capsules are modelled by means of a boundary integral technique to compute the flows, coupled to a finite element method to calculate the force exerted by the membranes on the fluids. We follow the motion and deformation of the capsules as they are convected towards each other after a sudden start of the flow. Our main finding is that, depending on their initial position and deformability, the two capsules may oscillate slowly about the flow gradient axis, get nearer to each other at each oscillation to finally interact strongly and separate. This minuet motion had not been identified previously. We identify the regions of space where either simple crossing or minuet occurs. This phenomenon has a marked influence on the irreversible trajectory drift of two capsules after crossing: the minuet process leads to a significant trajectory displacement along the flow gradient when none was expected, based on the previous studies where the two capsules had a significant relative velocity.

Key words: capsule/cell dynamics, suspensions, boundary integral methods

\section{Introduction}

The hydrodynamics of pairwise interaction of deformable particles is a crucial topic for semi-dilute suspension rheology (Batchelor \& Green 1972a; Guazzelli \& Morris 2012). When the particles are deformable, their shear induced deformation leads to non-Newtonian and to self-diffusion effects. This has been demonstrated for liquid droplets (Loewenberg \& Hinch 1997; Guido \& Simeone 1998). The case of capsules

$\dagger$ Email address for correspondence: anne-virginie.salsac@utc.fr 
(liquid drop enclosed by a thin elastic membrane) is particularly complex because the motion and deformation of those particles result from nonlinear fluid-structure interactions that are difficult to model. For example, the capsules may be highly deformed as they cross each other, which leads to the formation of a thin liquid film between the two particles and to potential damage of the membrane due to high shearing forces.

This phenomenon is usually studied in a simple shear flow with velocity components given by $v_{1}^{\infty}=\dot{\gamma} x_{2}, v_{2}^{\infty}=v_{3}^{\infty}=0$ in a laboratory Cartesian reference frame, where $\dot{\gamma}$ is the shear rate. The two capsules $C_{1}$ and $C_{2}$ are initially positioned with distances $\Delta X_{1}^{(0)}, \Delta X_{2}^{(0)}, \Delta X_{3}^{(0)}$ between their centres. The first three-dimensional model of two initially spherical identical capsules (radius $a$ ) interacting in simple shear flow is due to Lac, Morel \& Barthès-Biesel (2007), who considered the case where the two capsules had their centres in the same $x_{1} x_{2}$ shear plane $\left(\Delta X_{3}^{(0)}=0\right)$. The capsule membrane is treated as a very thin sheet of a hyperelastic material devoid of bending resistance and the flow Reynolds number is assumed to be negligible. Lac et al. showed that, in a reference frame centred on $C_{1}$, capsule $C_{2}$ is first displaced along the velocity gradient so that it can overpass ('jump over') $C_{1}$ and it is then shifted back towards the flow axis as it moves away. However, the final separation $\Delta X_{2}^{(f)}$ is larger than the initial one $\Delta X_{2}^{(0)}$. The crossing thus leads to an irreversible trajectory shift along the shear gradient ( $x_{2}$-direction). This effect, which decreases with an increase of the capsule deformability and/or the initial distance $\Delta X_{2}^{(0)}$, ultimately leads to self-diffusion effects in a suspension. We propose to call this crossing process the leapfrog motion. The same situation was later considered where the two spherical capsules were replaced by two red blood cells (Omori et al. 2013) or two vesicles (Gires et al. 2014). In both instances, it is found that the particles do a leapfrog motion with a trajectory shift that evolves qualitatively, as found previously by Lac et al. Experimental measurements of the trajectory of liquid filled giant lipid vesicles compare well with the predictions of the flow model (Kantsler, Segre \& Steinberg 2008; Gires et al. 2014). In all the aforementioned studies, the flow field around the capsules was computed by means of the boundary integral representation of the Stokes equations. As a consequence, the pair of capsules is effectively interacting in an infinite flow domain.

Finite differences and front tracking techniques can also be used to study the interaction problem: the advantage is that non-Newtonian or finite inertia effects in the suspending fluid can be considered. However, the computation is then usually performed in a flow domain, bounded by two walls parallel to the $x_{1} x_{3}$-plane where the velocity is imposed (to create the simple shear flow). On the other boundaries of the box, periodic flow conditions are imposed. Doddi \& Bagchi (2008) used this technique to model the pair interaction of two initially spherical capsules, when the inertia of the flow was not negligible. They found that, when the flow Reynolds number increased, the capsules did not cross, but reversed their motion. This phenomenon was confirmed for a pair of liquid droplets (Olapade, Singh \& Sarkar 2009). However, the spiralling motion reported by Doddi and Bagchi, is linked to the size of their computational domain and is a confinement effect. Indeed, for the results to be independent of the computational procedure and to be transposable to unbounded flow situations, the domain has to be large enough, typically $40 a \times 10 a \times 5 a$, for in-shear-plane crossing (Olapade et al. 2009). Pranay et al. (2010) considered the case where the suspending fluid is a dilute polymeric solution. They found that the presence of polymer leads to a decrease of the trajectory shift only when the capsule deformability is low. A recent study (Singh \& Sarkar 2015) considered the pair interaction of two capsules with different deformability 
and found that the trajectory shift is influenced by the stiffness ratio. All the studies based on finite difference and front tracking techniques only considered pairs of capsules with their centroids in the same shear plane, where they remain because of the problem symmetry.

However, in a suspension, two nearby capsules will not necessarily have their centres of mass in the same shear plane. For example, special care has to be taken in vesicle experiments to ensure that this is approximately the case. Accordingly, Lac \& Barthès-Biesel (2008) also modelled the three-dimensional motion of two capsules positioned in two different shear planes $\left(\Delta X_{3}^{(0)} \neq 0\right)$. In this case, a sideways leapfrog motion occurs with a maximum trajectory displacement along both the $x_{2}$ and $x_{3}$-directions, which decreases as $\Delta X_{3}^{(0)}$ and/or capsule deformability increase. Lac and Barthès-Biesel also showed that the final trajectory shift along the $x_{3}$-axis is indeed smaller than the one along the velocity gradient $x_{2}$-axis (approximately one third), but present nevertheless. This was also globally confirmed by Gires et al. (2014) for vesicles.

However, in all aforementioned studies, the two capsules always had a significant initial relative velocity, obtained by means of $\Delta X_{2}^{(0)} \geqslant 0.5 a$. This choice was made to avoid very long computations. The only exceptions are due to Lac et al. (2007) and Omori et al. (2013), who showed that, for two capsules located on the same streamline $\left(\Delta X_{1}^{(0)} \neq 0, \Delta X_{2}^{(0)}=\Delta X_{3}^{(0)}=0\right)$, the perturbation created by the deformation and membrane rotation of the two capsules created a small but finite velocity field that displaced the centroids along the $x_{2}$-axis and led to a relative velocity of the capsules and to a leapfrog motion. The result was interesting as it showed that such initial conditions led to the largest self-diffusion effect. The conclusion of this review is that there is presently no information on the interaction of two capsules when their centres are located in the $x_{1} x_{3}$-plane.

The objective of this paper is to fill this gap and to investigate the three-dimensional motion of two capsules when their centroids are in or near the same $x_{1} x_{3}$-plane. We will take advantage of the computational technique that we have developed, based on the coupling of a boundary integral to compute the flow and finite elements to compute the capsule wall mechanics (Walter et al. 2010). This coupling has proved to be very stable in a number of situations where long transient motion of a single capsule needed to be monitored (Walter, Salsac \& Barthès-Biesel 2011; Hu, Salsac \& Barthès-Biesel 2012; Dupont, Salsac \& Barthès-Biesel 2013; Dupont et al. 2016). We will see that a new interaction mode is revealed: given the choice, the capsules oscillate around the shear gradient axis, rather than around the vorticity axis. We call this interaction mode the minuet motion, as it is similar to the one reported for Volvox algae (Drescher et al. 2009), albeit for different hydrodynamic interactions.

The paper is organized as follows: the problem is set out in $\S 2$ together with a short description of the numerical method. The different types of capsule interaction are presented in $\S 3$, where we also discuss the main factors that determine the motion type. In $\S 4$, we analyse which factors determine the motion type and illustrate the area of space where oscillatory motion is expected to occur. In $\S 5$, we then study the consequences on the trajectory shift and self-diffusion phenomena in the suspension. In the final $\S 6$, we summarize the findings and provide a conclusion.

\section{Problem statement and numerical method}

\subsection{Problem description}

Two identical spherical capsules $C_{1}$ and $C_{2}$ (radius $a$ ), filled with a Newtonian liquid (viscosity $\mu$, density $\rho$ ) and enclosed by a very thin hyper-elastic membrane (surface 


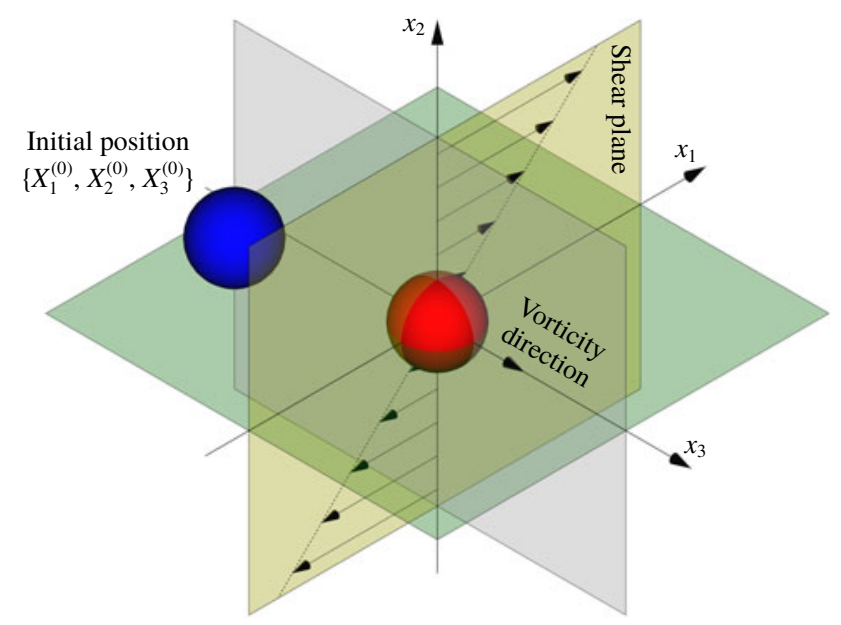

Figure 1. Two capsules flowing in simple shear flow. The reference frame is linked to capsule $C_{1}$ and the coordinate system is centred on it. The centre of $C_{2}$ is initially positioned at $\boldsymbol{X}^{(0)}$.

shear modulus $G_{s}$ and area dilation modulus $K_{s}$ ), are freely suspended in another Newtonian liquid (viscosity $\mu$, density $\rho$ ) and subjected to a simple shear flow with shear rate $\dot{\gamma}$. Inertia effect is neglected. The capsules centroids are denoted $G_{1}$ and $G_{2}$. We use a reference frame centred on $G_{1}$, that moves with it (figure 1). Our objective is to study the interaction process between the two capsules as they are convected by the flow and, specifically, to compute the evolution of the velocity $\boldsymbol{V}(t)$ and position $\boldsymbol{X}(t)$ of $G_{2}$ with time $t$.

The undisturbed flow $\boldsymbol{v}^{\infty}$ of the external liquid is given by

$$
v_{1}^{\infty}(\boldsymbol{x})=\dot{\gamma} x_{2} ; \quad v_{2}^{\infty}(\boldsymbol{x})=v_{3}^{\infty}(\boldsymbol{x})=0 .
$$

Note that, since the problem is inertialess, the flow field has the same expression (2.1) in a laboratory reference frame. The motion of the internal and external fluids is governed by the Stokes equations, with associated boundary conditions given by:

(i) vanishing flow perturbation far from the capsules:

$$
\boldsymbol{v}^{e x t}(\boldsymbol{x}) \rightarrow \boldsymbol{v}^{\infty}(\boldsymbol{x}) \quad \text { as }\left\|\boldsymbol{x}-\boldsymbol{x}\left(G_{\alpha}\right)\right\| \rightarrow \infty \quad \alpha=1,2 ;
$$

(ii) at a material point $\boldsymbol{x}$ located on the membrane of either capsule

$$
\begin{gathered}
\boldsymbol{v}^{e x t}(\boldsymbol{x})=\boldsymbol{v}^{i n t}(\boldsymbol{x})=\dot{\boldsymbol{x}}, \\
\boldsymbol{q}+\nabla_{s} \cdot \boldsymbol{T}=\mathbf{0},
\end{gathered}
$$

where the superscript ext or int refers respectively to the suspending fluid or to the capsule internal liquid. The jump of viscous traction across the membranes is $\boldsymbol{q}$, the in-plane elastic tension tensor in the membrane is denoted $\boldsymbol{T}$ and $\nabla_{s}$ is the surface gradient operator. Equation (2.4) is the membrane equilibrium equation, which expresses the dynamic coupling between the solid membranes and the fluids.

As this fluid-structure interaction problem is now classical, we will only present the main hypotheses used here and refer the reader to the comprehensive review of 
Barthès-Biesel (2016). The fluid velocity at any point $\boldsymbol{x}$ is written as a boundary integral on the surfaces $S_{1}$ and $S_{2}$ of the two capsules (Lac et al. 2007)

$$
\boldsymbol{v}(\boldsymbol{x})=\boldsymbol{v}^{\infty}(\boldsymbol{x})-\frac{1}{8 \pi \mu} \int_{S_{1} \cup S_{2}} \boldsymbol{J}(\boldsymbol{x}, \boldsymbol{y}) \cdot \boldsymbol{q}(\boldsymbol{y}) \mathrm{d} S(\boldsymbol{y}),
$$

where $\boldsymbol{J}$ is the free space Green's function given by

$$
J(x, y)=\frac{I}{\|x-y\|}+\frac{(x-y) \otimes(x-y)}{\|x-y\|^{3}},
$$

where $\boldsymbol{I}$ is the identity tensor. The pressure $p-p_{0}$ at a point $\boldsymbol{x}$ is given by

$$
p(\boldsymbol{x})-p_{0}=-\frac{1}{4 \pi} \int_{S_{1} \cup S_{2}} \frac{\boldsymbol{q}(\boldsymbol{y}) \cdot(\boldsymbol{x}-\boldsymbol{y})}{\|\boldsymbol{x}-\boldsymbol{y}\|^{3}} \mathrm{~d} S(\boldsymbol{y}),
$$

where $p_{0}$ denotes the far field pressure.

The capsule membrane is assumed to be an infinitely thin sheet of a threedimensional isotropic volume incompressible material that satisfies a neo-Hookean (NH) constitutive law. Bending resistance is neglected. The membrane constitutive law relates the principal elastic tensions (forces per unit arclength measured in the membrane plane) $T_{1}$ and $T_{2}$ to the two principal extension ratios $\lambda_{1}$ and $\lambda_{2}$

$$
T_{1}=\frac{G_{s}}{\lambda_{1} \lambda_{2}}\left[\lambda_{1}^{2}-\frac{1}{\left(\lambda_{1} \lambda_{2}\right)^{2}}\right],
$$

with a similar expression for $T_{2}$, where the subscripts 1 and 2 are permuted. For simplicity, we have denoted these principal directions 1 and 2 , but they should not be confused with the Cartesian directions in space. The surface shear elastic modulus $G_{s}$ and area dilation modulus $K_{s}$ are related by $K_{s}=3 G_{s}$ (Barthès-Biesel 2016). The corresponding total deformation energy of the membrane of one capsule is given by

$$
W=\frac{G_{s}}{2} \int_{S}\left(\lambda_{1}^{2}+\lambda_{3}^{2}-3+\frac{1}{\lambda_{1}^{2} \lambda_{2}^{2}}\right) \mathrm{d} S,
$$

where $S$ stands for either $S_{1}$ or $S_{2}$.

The main parameters are the capillary number

$$
C a=\frac{\mu \dot{\gamma} a}{G_{s}},
$$

which measures the relative stiffness of the capsule, and the initial position of $G_{2}$ at time $t=0$, when the flow is suddenly started

$$
\boldsymbol{X}(0)=\boldsymbol{X}^{(0)}=\left\{X_{1}^{(0)}, X_{2}^{(0)}, X_{3}^{(0)}\right\} .
$$

We shall discuss the typical case where $X_{1}^{(0)}$ and $X_{3}^{(0)}$ are negative while $X_{2}^{(0)}$ is positive: the flow of $G_{2}$ occurs from left to right in the trajectory figures. The case $X_{3}^{(0)}>0$ provides the same results as the typical case since it corresponds to the symmetric configuration with respect to the shear plane. Positive values of $X_{1}^{(0)}$ and negative ones for $X_{2}^{(0)}$ correspond to the mirror image of the typical case, with respect to the $x_{2} x_{3}$-plane. When the crossing process is completed, the final steady position of $G_{2}$ is $\left\{X_{1}^{(f)}, X_{2}^{(f)}, X_{3}^{(f)}\right\}$. We define the final trajectory shifts of the capsule

$$
\delta_{2}=\left|X_{2}^{(f)}\right|-\left|X_{2}^{(0)}\right|, \quad \delta_{3}=\left|X_{3}^{(f)}\right|-\left|X_{3}^{(0)}\right|,
$$

which are computed for $\left|X_{1}^{(f)}\right|=10 a$. 


\subsection{Numerical method}

The fluid-structure interaction problem is solved by means of the numerical scheme that couples a boundary integral method (BI) to solve the fluid flow and the finite element method (FE) to solve the membrane mechanics (Walter et al. 2010; Hu et al. 2012). This method is well adapted to Stokes flows and has the advantage of requiring the discretization of the capsule surfaces $S_{1}$ and $S_{2}$ only. It automatically accounts for an unbounded fluid domain. The model inputs are the capillary number $\mathrm{Ca}$ and the initial position $\boldsymbol{X}^{(0)}$ of capsule $C_{2}$. Following Lac et al. (2007), we use the fact that the two capsules are identical to centre the flow field on the midpoint $O$ of $G_{1} G_{2}$, so that (2.5) can be solved on only one capsule and becomes

$$
\boldsymbol{v}(\boldsymbol{x})=\boldsymbol{v}^{\infty}(\boldsymbol{x})-\frac{1}{8 \pi \mu} \int_{S_{2}}[\boldsymbol{J}(\boldsymbol{x}, \boldsymbol{y})-\boldsymbol{J}(\boldsymbol{x},-\boldsymbol{y})] \cdot \boldsymbol{q}(\boldsymbol{y}) \mathrm{d} S(\boldsymbol{y}) .
$$

The capsule surface $S_{2}$ is discretized using $P_{2}$ triangle elements, in which 6 nodes are allocated at the vertices and the middle of each side. The mesh is generated from the initial spherical shape by projecting a regular icosahedron to the sphere and then subdividing each element subsequently to the desired precision. A mesh of 1280 elements and 2562 nodes has been used in all the simulations, corresponding to a characteristic mesh size $\Delta h_{c}=O(0.1 a)$. Such a spatial discretization has been shown to lead to a relative error of order $10^{-3}$ on the Taylor deformation of a single capsule in shear flow (Walter et al. 2010). Furthermore, it can withstand some membrane compression without creating any numerical instability (Hu et al. 2012). The explicit time iteration is stable only if the time step is such that $\dot{\gamma} \Delta t<O\left(\mathrm{Ca}_{\Delta} h_{c} / a\right)$ (Walter et al. 2010). In the case of a NH membrane and $C a=0.3, \dot{\gamma} \Delta t=5 \times 10^{-4}$ allows us to compute the capsule trajectory over long times without stability issues. We stop the computation when the distance $G_{1} G_{2}$ is larger than $10 a$.

At time $t=0, C_{2}$ (positioned at $\left\{X_{1}^{(0)} / 2, X_{2}^{(0)} / 2, X_{3}^{(0)} / 2\right\}$ ) and $C_{1}$ (positioned at $\left.\left\{-X_{1}^{(0)} / 2,-X_{2}^{(0)} / 2,-X_{3}^{(0)} / 2\right\}\right)$ are subjected to the sudden start of the flow. The model follows the motion of the capsule membrane over time. At any time, the model can thus output the position of the surface nodes, from which it is possible to infer, for each capsule, the deformation and elastic tensions in the membrane as well as the position and velocity of the centroid. The knowledge of this velocity allows us to transcript the results in the reference frame linked to $C_{1}$.

The accuracy of the numerical model is checked by comparing capsules trajectories with those obtained by Lac et al. (2007) and Lac \& Barthès-Biesel (2008), and also by assessing the influence of the time step and of the spatial discretization of the capsule membranes (see the Appendix): the conclusion is that the centroid trajectories are accurate to within $0.05 a$.

\section{Types of capsule interaction: simple crossing (leapfrog) or minuet motion}

A major finding of our work is that, depending on the initial position of $C_{2}$, there are two types of motion:

(i) Simple crossing (also denoted leapfrog): $C_{2}$ catches up with $C_{1}$, interacts and goes away. The two capsules are not necessarily in the same shear plane.

(ii) Minuet motion: $C_{2}$ catches up with $C_{1}$, interacts, overpasses $C_{1}$, reverses its motion and repeats the process one, two or three times before getting away.

The two motions are illustrated and analysed in the following. 
(a)

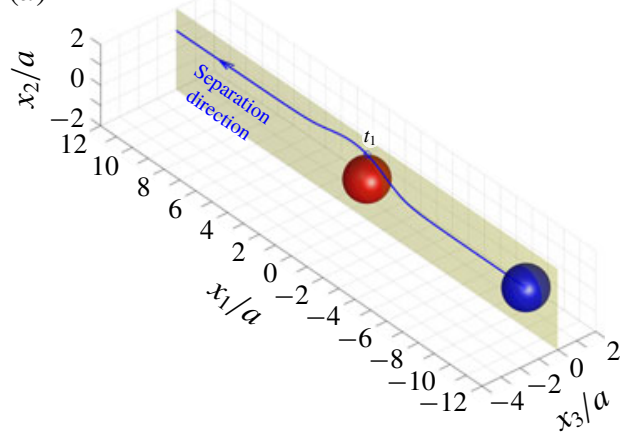

(c)

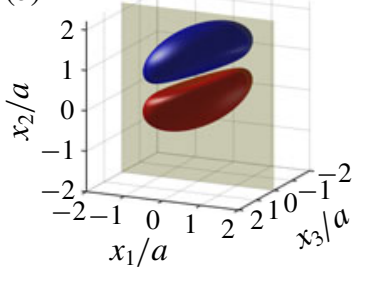

(d)

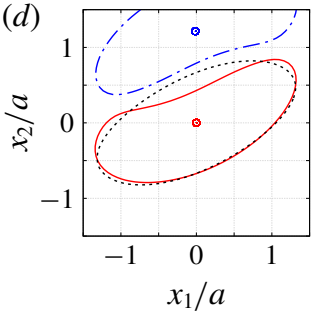

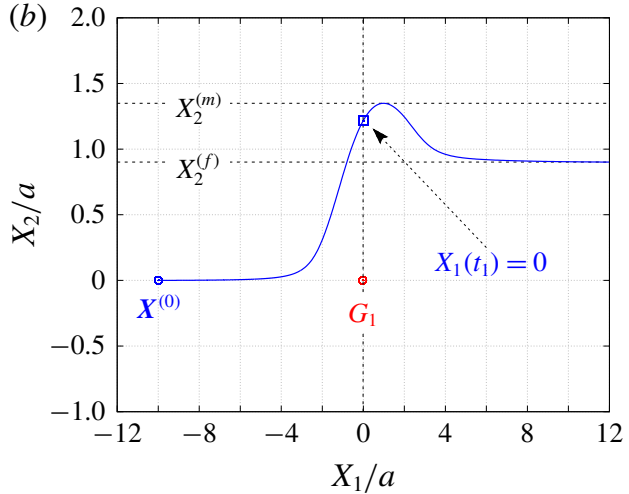

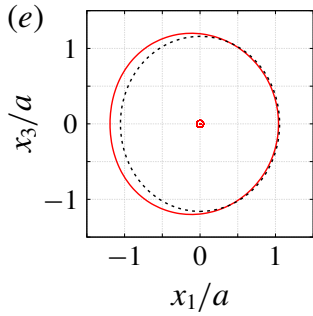

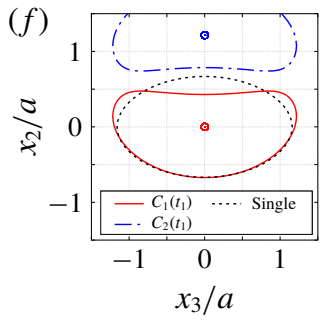

FIGURE 2. Leapfrog motion of two capsules with their centres in the same shear plane $\left(X_{1}^{(0)} / a=-10, X_{2}^{(0)}=0, X_{3}^{(0)}=0, C a=0.3\right)$. (a) Three-dimensional view of the trajectory of $C_{2}$. (b) $G_{2}$ trajectory in the shear plane. (c) Three-dimensional view of the deformed capsules at $t_{1}$ when $X_{1}\left(t_{1}\right)=0 .(d-f) C_{1}$ intersections with the three coordinates planes at $t_{1}$.

\subsection{Single interaction: leapfrog motion}

As a reference, we consider the situation where the two capsules are in the shear plane on the same streamline $\left(X_{1}^{(0)}=10 a, X_{2}^{(0)}=0, X_{3}^{(0)}=0, C a=0.3\right)$. Similar computations have been made by Lac et al. (2007) for pre-inflated capsules, but only the value of the final trajectory shift is reported. Under Stokes flow conditions, the capsules must remain in this plane. The trajectory of $C_{2}$ is shown in figure 2(a,b) (movie 1 available at https://doi.org/10.1017/jfm.2020.181).

When the flow is started, the two capsules are far enough from each other that they behave as if they were almost alone in the fluid. Within $\dot{\gamma} t \sim 6$ they reach a roughly ellipsoidal shape around which the membrane rotates, as shown in figure $2(d)$ (see the review by Barthès-Biesel (2016)). The deformation and rotation of $C_{1}$ lead to a stresslet that creates a small velocity field and a small depression. This perturbation displaces $C_{2}$ along the velocity gradient in the direction that moves it towards $C_{1}$, as shown in figure $2(b)$. Note that this phenomenon is the opposite of the one observed by Doddi \& Bagchi (2008) when inertia is taken into account. As a consequence, $V_{2}(t)>0$ for $t>0$ and $C_{2}$ is convected towards $C_{1}$, as shown in figure $2(a, b)$. The only way for $C_{2}$ to pass $C_{1}$ is to 'jump' over it in the $x_{1} x_{2}$-plane (thus the term 'leapfrog motion'). The fact that the motion is constrained to the shear plane leads to a high velocity difference $\left\|\boldsymbol{V}-\boldsymbol{v}^{\infty}\right\| / \dot{\gamma} a$ in the $x_{1}$ - and $x_{2}$-directions (figure 3). The evolution of the pressure at the midpoint between $G_{1}$ and $G_{2}$ (2.7) can also explain the interaction phenomenon: indeed, as $\left|X_{1}(t) / a\right|$ decreases, the pressure in 

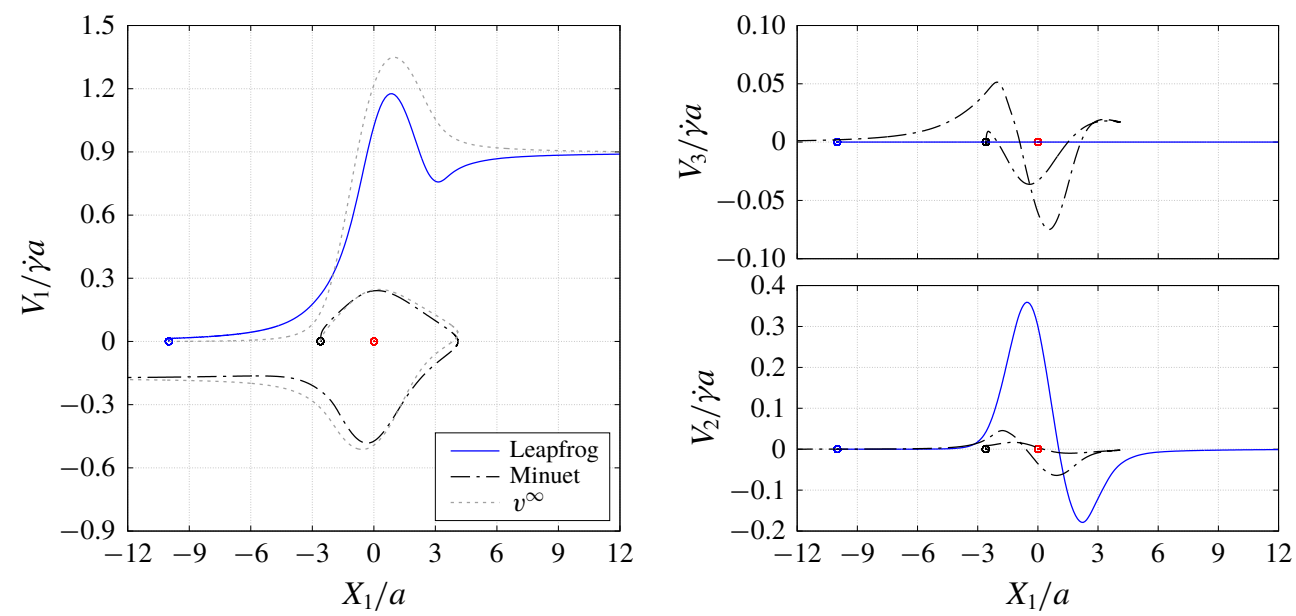

FIGURE 3. Evolution of $C_{2}$ velocity during a leapfrog $\left(X_{1}^{(0)} / a=-10, X_{2}^{(0)}=X_{3}^{(0)}=0\right)$ or minuet $\left(X_{1}^{(0)} / a=X_{3}^{(0)} / a=-2.6, X_{2}^{(0)}=0\right)$ for $C a=0.3$.
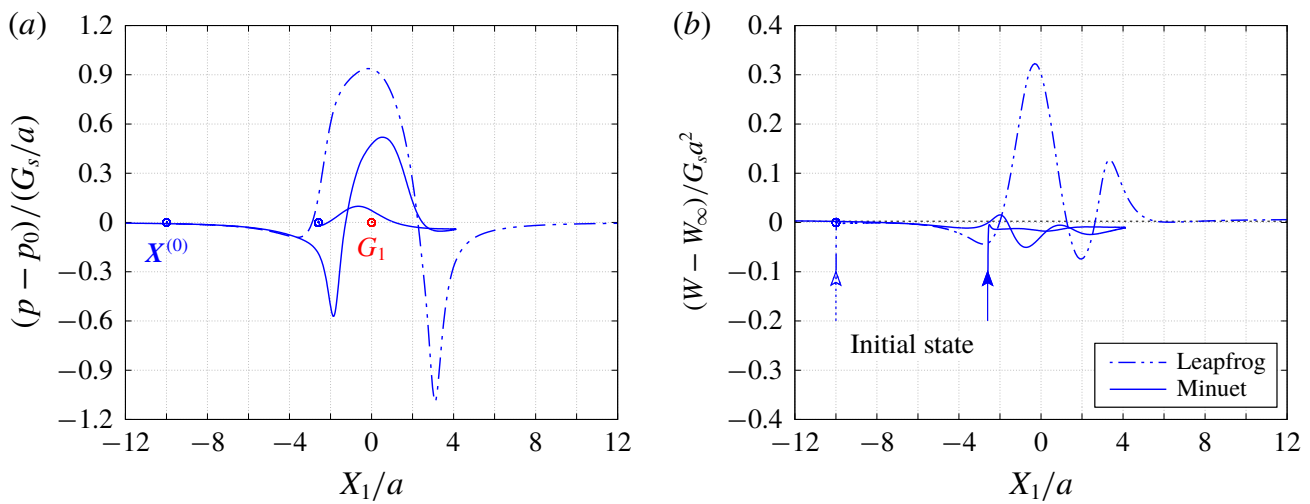

FIgURE 4. Pressure and membrane energy evolution during the leapfrog and minuet motions for $C a=0.3$. (a) Pressure $p$ at the mid-point between $G_{1}$ and $G_{2} ;(b)$ membrane elastic energy $W$. Leapfrog: $X_{1}^{(0)} / a=-10, X_{2}^{(0)}=0, X_{3}^{(0)}=0$. Minuet: $X_{1}^{(0)} / a=-2.6$, $X_{2}^{(0)}=0, X_{3}^{(0)} / a=-2.6$.

the lubrication film between the two capsules increases (figure $4 a$ ) and pushes $C_{2}$ in the $x_{2}$-direction, along which the maximum displacement of $G_{2}$ is $X_{2}^{(m)}$. As $C_{2}$ overtakes $C_{1}$, the widening of the lubrication film leads to a depression (figure $4 a$ ), which decreases $X_{2}$, until a final steady value $X_{2}^{(f)}$ is reached when the two capsules are far apart $\left(\left|X_{1}\right| \geqslant 6 a\right.$, in this case). The final trajectory shift $\delta_{2}=\left|X_{2}^{(f)}-X_{2}^{(0)}\right|$ is $0.9 a$, and is equal to the one found by Lac et al. under the same flow situation for a $5 \%$ pre-inflation. The finite value of $\delta_{2}$ indicates that the two capsule interaction leads to self-diffusion effects in a dilute suspension of capsules. The deformed profiles of $C_{1}$ (equivalently of $C_{2}$ ) in the $x_{1} x_{2}-, x_{1} x_{3^{-}}$and $x_{2} x_{3}$-planes are shown in figure $2(d-f)$ at time $\dot{\gamma} t_{1}=9.7$, when the two capsules cross, which we define by $X_{1}\left(t_{1}\right)=0$. Figure $2(d, f)$ shows that there is indeed a thin lubrication film between 
(a)

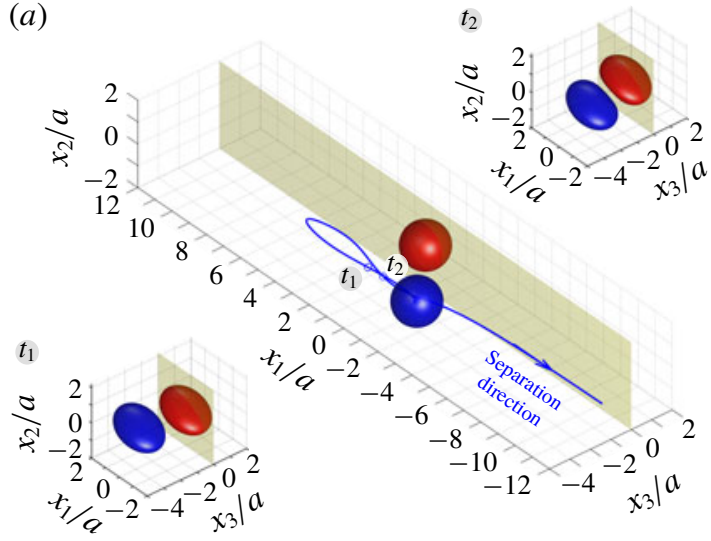

(b)
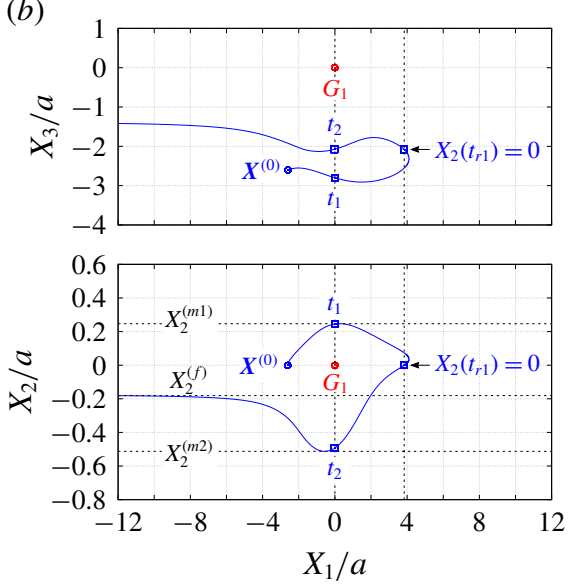

(c)

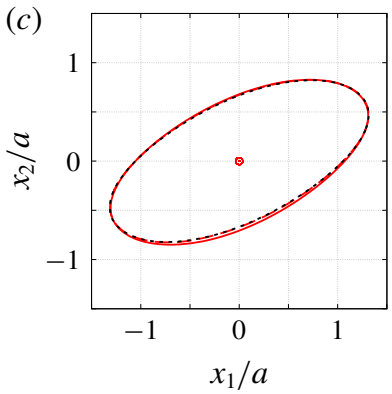

(d)

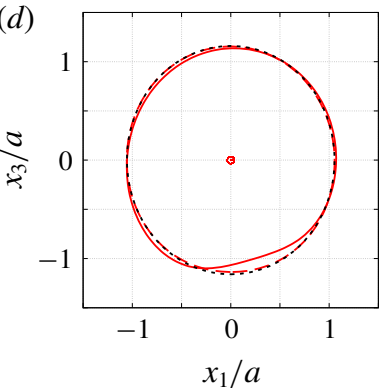

(e)

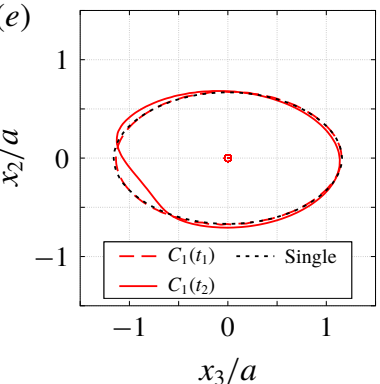

Figure 5. Minuet of two capsules $\left(X_{1}^{(0)} / a=-2.6, X_{2}^{(0)}=0, X_{3}^{(0)} / a=-2.6, C a=0.3\right)$. (a) Three-dimensional view of the trajectory of $C_{2}$. (b) Projections of $G_{2}$ trajectories in the $x_{1} x_{2}$ - and $x_{1} x_{3}$-planes. $(c-e) C_{1}$ profile intersections with the three coordinates planes at times $t_{1}$ and $t_{2}$ when $X_{1}\left(t_{1}\right)=X_{1}\left(t_{2}\right)=0$.

the two capsules (which corroborates the pressure build-up) and that the two capsules are highly deformed. The global deformation can be assessed through the membrane elastic energy $W$ given by (2.9). The value of $W\left(X_{1}\right)-W_{\infty}$, where $W_{\infty}$ is the deformation energy of a single capsule, allows us to estimate the intensity of the mechanical interaction between the two capsules. During the close interaction, the two capsules undergo large transient deformation, leading to a peak in $W-W_{\infty}$ (figure $4 b$ ). The separation process leads to some deformation oscillations, until a final steady state is reached, which is identical to the single capsule one, elastic energy wise (figure $4 b$ ).

\subsection{Minuet motion}

We now consider the case $X_{1}^{(0)} / a=-2.6, X_{2}^{(0)}=0, X_{3}^{(0)} / a=-2.6, C a=0.3$, where capsule $C_{2}$ is located off the shear plane and can thus move freely in space: this leads to trajectories that are completely different from the ones described above. As in the previous case, the rotation of $C_{1}$ displaces $G_{2}$ along the velocity gradient, so that $C_{2}$ is convected towards $C_{1}$. The three-dimensional trajectory of $C_{2}$ is shown in figure $5(a)$, where the two capsules are shown in their initial positions (movie 2). In order to get a clearer grasp of the process, we analyse the trajectories of the projections of 
$G_{2}$ in the $x_{1} x_{2}$ - and $x_{1} x_{3}$-planes in figure $5(b)$. As $\left|X_{1}(t) / a\right|$ decreases, the pressure increases slightly (figure $4 a$ ): this leads to a slight increase in both $\left|X_{2}(t)\right|$ and $\left|X_{3}(t)\right|$ (figure $5 b$ ), which allows enough space for capsule $C_{2}$ to pass $C_{1}$ by moving around the $x_{2}$-axis (insets in figure $5 a$ ). Correspondingly, the maximum displacement $\left|X_{2}^{(m 1)}\right|$ is smaller than in the leapfrog situation. Similarly, the velocity difference $\left\|\boldsymbol{V}-\boldsymbol{v}^{\infty}\right\| / \dot{\gamma} a$ remains small while occurring in all three directions, as shown in figure 3 . The energy variation $W-W_{\infty}$ is also very small (figure $4 b$ ), which indicates that there is little mechanical interaction between the capsules. This point is further corroborated by the quasi-superposition of the deformed profiles of $C_{1}$ at time $t_{1}$ when $X_{1}\left(t_{1}\right)=0$, and when it is alone in the flow (figure $5 c-e$ ).

As the capsules separate, the small depression (figure $4 a$ ) leads to a negative displacement along the $x_{2}$-axis, which takes $G_{2}$ into the reverse flow region and entices $C_{2}$ to move back towards $C_{1}$. The pressure is still negative when the reversal takes place, so that $\left|X_{3}(t)\right|$ decreases. As a consequence, there is not enough space for $C_{2}$ to move around $C_{1}$ in a $x_{1} x_{3}$-plane and a sideways leapfrog motion takes place, which is qualitatively similar to the one that occurs in the shear plane, as described in the previous section: there is a significant pressure build-up in the lubrication film, followed by a depression as the capsules part (figure $4 a$ ). The pressure variation leads to an increase of $\left|X_{2}(t)\right|$ up to a value $\left|X_{2}^{(m 2)}\right|$, which is large enough to allow the further decrease to the final displacement $\left|X_{2}^{(f 2)}\right|$, without crossing into a reverse flow region. At time $t_{2}$ when $X_{1}\left(t_{2}\right)=0$, the two capsules are closer than at time $t_{1}$ and thus undergo a transient deformation, as appears in figure 5(a) (inset) and in figure $5(d, e)$. Correspondingly, the mechanical energy $W-W_{\infty}$ undergoes a transient variation, which, however, is much smaller than the one that occurs when the capsules cross in the shear plane (figure $4 b$ ). We deduce that minuet motion is less energy consuming than leapfrog motion.

If we now start with the same initial conditions $\left(X_{1}^{(0)} / a=-2.6, X_{2}^{(0)}=0, X_{3}^{(0)} / a=\right.$ -2.6) but reduce the capsule deformability by setting $C a=0.2$, two reversals occur, as shown in figure 6 (movies 3 and 4). The first reversal is essentially the same as for $C a=0.3$. However, when the capsules cross again at time $t_{2}$, there is still enough space to allow crossing around the $x_{3}$-axis. Furthermore, the displacement along the $x_{2}$-axis is still small $\left(X_{2}\left(t_{2}\right) / a=-0.39\right)$, compared to the one observed for $C a=0.3\left(X_{2}\left(t_{2}\right) / a=-0.51\right)$, so that the pressure induced trajectory shift forces $G_{2}$ to cross again into the reverse flow region: the capsule is thus convected again towards $C_{1}$. During the third crossing, since $\left|X_{3}\left(t_{3}\right)\right| / a<2$, the capsule has to do a sideways leapfrog motion that leads to a displacement $X_{2}\left(t_{2}\right) / a=0.92$, that is large enough to accommodate the trajectory shift without changing the flow direction of the capsule: the latter finally goes back in the direction where it came from. Note that, at time $t_{3}$, the two capsules undergo a significant transient deformation, due to a strong interaction. This phenomenon is unexpected in view of the fairly large initial distance between the capsules.

\section{What factors determine the motion type?}

The type of motion (leapfrog or minuet) is determined by the evolution of $X_{2}(t)$ after crossing, since it is a change of sign of $X_{2}(t)$ that causes reversal of motion. At crossing, the film pressure displaces $G_{2}$ along the velocity gradient, to a maximum value $\left|X_{2}^{(m)}\right|$. After crossing, the separation process and the subsequent depression lead to a displacement $\Delta_{2}$ of $G_{2}$ back to the $x_{1}$-axis. For leapfrog motion, the trajectory shift is easily identified as $\Delta_{2}=\left|X_{2}^{(m)}-X_{2}^{(f)}\right|$ (see figure $2 b$ ). When reversal occurs, the 

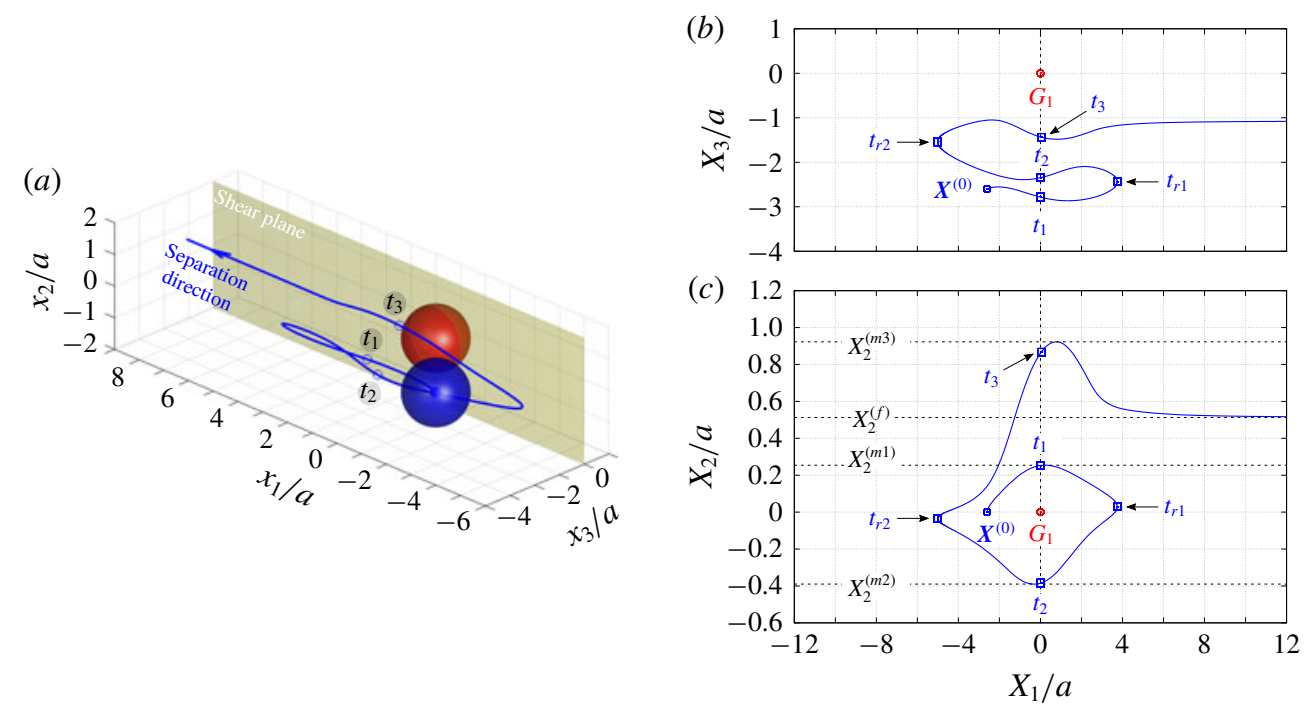

$(d)$

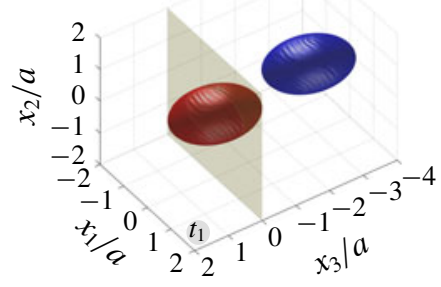

$(e)$

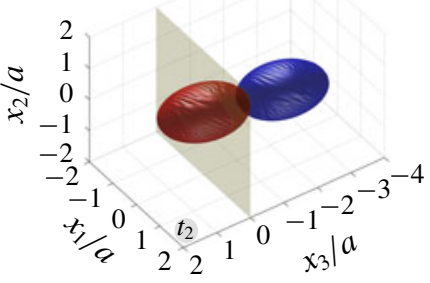

$(f)$

FIGURE 6. Minuet with multiple reversals for $X_{1}^{(0)} / a=-2.6, X_{2}^{(0)}=0, X_{3}^{(0)} / a=-2.6$, $C a=0.2$. The instants of close interaction when $X_{1}=0$ are denoted $t_{1}, t_{2}$ and $t_{3}$ in chronological order. (a) Three-dimensional view of the trajectory of $C_{2}$. $(b, c)$ Projections of $G_{2}$ trajectories in the $x_{1} x_{2}$ and $x_{1} x_{3}$-planes. $(d)$ Three-dimensional profiles at close interaction.

film pressure decrease also leads to a trajectory shift $\Delta_{2}$, which is difficult to evaluate in a simple fashion. However, since $\Delta_{2}$ is a consequence of the separation process, we can surmise that it follows an evolution similar to the one found in leapfrog motion in the shear plane, i.e. that it decreases when the capsule separation $\left|X_{2}^{(0)}\right|$ and/or $\left|X_{3}^{(0)}\right|$ increase and when the capsule deformability $C a$ increases (Lac et al. 2007; Lac \& Barthès-Biesel 2008; Pranay et al. 2010; Omori et al. 2013; Gires et al. 2014). We conclude that, whenever $\left|X_{2}^{(m)}\right|$ is less than $\Delta_{2}$, reverse motion is to be expected. The main parameters that determine the values of $\left|X_{2}^{(m)}\right|$ and of $\Delta_{2}$, are the initial capsule separation $\boldsymbol{X}^{(0)}$ and the capillary number $\mathrm{Ca}$. We now study their influence separately.

\subsection{Effect of the initial capsule separation $\boldsymbol{X}^{(0)}$}

The effect of the initial offset $\left|X_{3}^{(0)}\right|$ from the shear plane is shown for $C a=0.3$, $X_{1}^{(0)} / a=-3.0$ and $X_{2}^{(0)} / a=0$ in figure 7 . When $\left|X_{3}^{(0)}\right|$ is small (e.g. $\left|X_{3}^{(0)} / a\right| \leqslant 2$ ), the situation is close to the one when the two capsules are in the same shear plane. Correspondingly, they undergo a sideways leapfrog motion with a displacement 


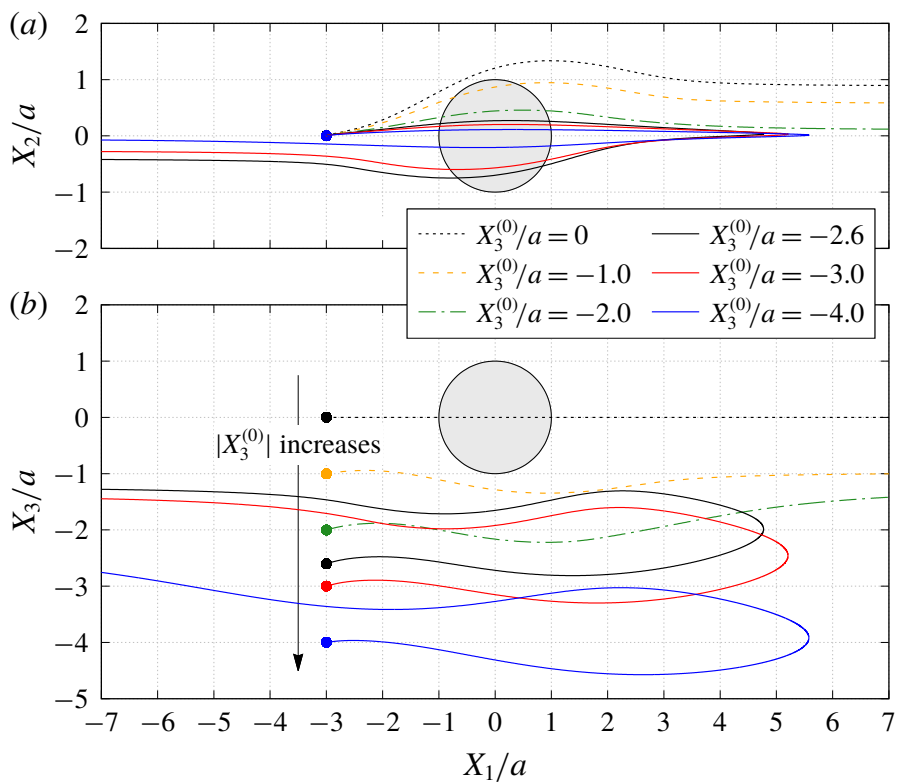

FIgURE 7. Effect of the initial position $X_{3}^{(0)}$ on the capsule trajectory for $C a=0.3$, $X_{1}^{(0)} / a=-3.0$ and $X_{2}^{(0)} / a=0$. Projections of $G_{2}$ trajectories in the $x_{1} x_{2}$-plane $(a)$ and in the $x_{1} x_{3}$-plane $(b)$. When $\left|X_{3}^{(0)}\right| / a \leqslant 2$, capsule $C_{2}$ has not enough room to move around $C_{1}$ : it overpasses it with a sideways leapfrog motion.

$\left|X_{2}^{(m)} / a\right| \geqslant 0.4$, which is large enough to allow direct crossing (figure $7 a$ ). For a larger offset $\left|X_{3}^{(0)} / a\right| \geqslant 2.6, C_{2}$ has room to move around $C_{1}$ in an $x_{1} x_{3}$-plane: then the displacement $\left|X_{2}^{(m)} / a\right| \sim 0.2$ is small (figure 7a), and thus reversal motion occurs during separation. As $\left|X_{3}^{(0)}\right|$ increases, the influence of $C_{1}$ decreases and the two capsules have almost no relative velocity. The motion shown in figure 7 for $\left|X_{3}^{(0)} / a\right|=4$ is near the limit of what can be reasonably computed: indeed, the capsule reaches $X_{1}=0$ at time $\dot{\gamma} t_{1}=50$ at the first crossing, and at time $\dot{\gamma} t_{2}=266$ at the second crossing. We conclude that the minuet motion is slow.

We now turn to the effect of the initial distance $\left|X_{1}^{(0)}\right|$ on the trajectory of $C_{2}$, as shown in figure 8 for $C a=0.3, X_{2}^{(0)} / a=0$ and $X_{3}^{(0)} / a=-2.6$. Note that, since $\left|X_{3}^{(0)} / a\right|>2, C_{2}$ has room to move around $C_{1}$ in an $x_{1} x_{3}$-plane. However, the far field perturbation created by $C_{1}$ is a stresslet, which varies as the square of the inverse distance $G_{1} G_{2}$. It is this perturbation that displaces $G_{2}$ along the $x_{2}$-axis and gives $C_{2}$ the small relative approach velocity, which leads to crossing. This perturbation velocity varies as $\left[X_{1}^{(0)} / a\right]^{-2}$ when $\left|X_{1}^{(0)}\right| / a \gg 1\left(\left|X_{1}^{(0)}\right| / a>4\right.$ in this particular case). Even though it is small, its prolonged effect over a long time leads to a significant $\left|X_{2}^{(m)}\right|$ displacement and thus to a sideways leapfrog motion. Note that the initial position $X_{2}^{(0)} / a=0$ is unstable when the capsules interact. However, when the initial distance $\left|X_{1}^{(0)}\right|$ becomes of order $a$ (e.g. $\left.\left|X_{1}^{(0)}\right| / a=3\right)$, the displacement $\left|X_{2}^{(m)}\right|$ has no time to build up and remains small: then reversal occurs.

Similar situations of capsule interaction have been considered by Lac \& BarthèsBiesel (2008), who did not report any motion reversal, even when they studied three- 

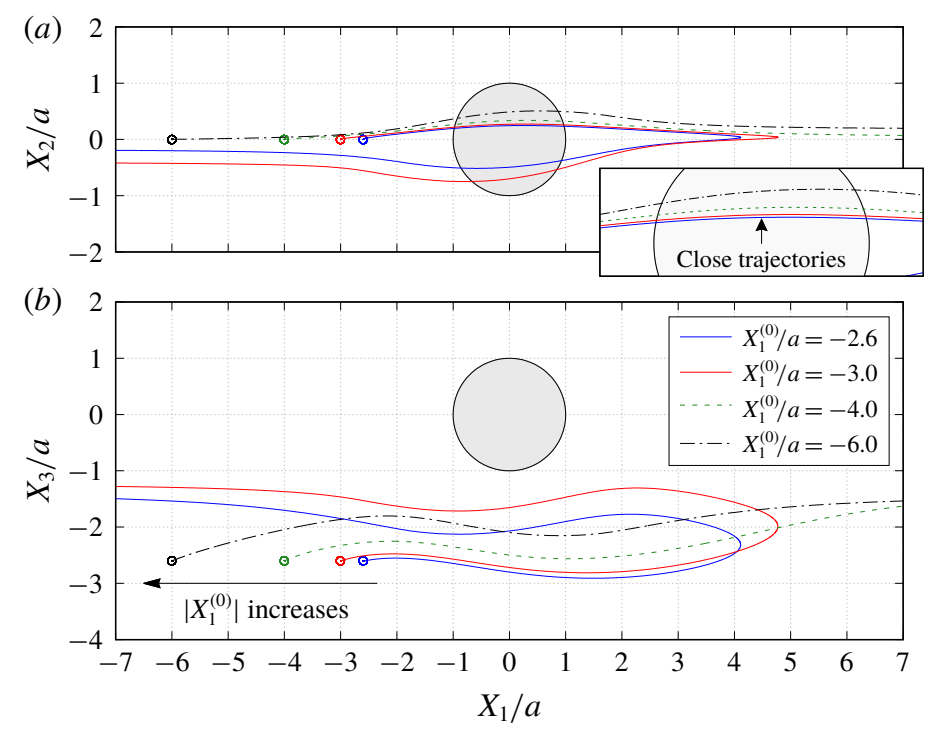

Figure 8. Effect of the initial position $X_{1}^{(0)}$ on the capsule trajectory for $C a=0.3$, $X_{2}^{(0)} / a=0$ and $X_{3}^{(0)} / a=-2.6$. Projections of $G_{2}$ trajectories in the $x_{1} x_{2}$-plane $(a)$ and in the $x_{1} x_{3}$-plane $(b)$. When $\left|X_{1}^{(0)}\right| / a \geqslant 4$, capsule $C_{2}$ has moved sufficiently across the streamlines that it can overpass $C_{1}$ with a sideways leapfrog motion.

dimensional motions with non-zero values of $X_{3}^{(0)}$. This is due to the fact that they started with $X_{2}^{(0)} / a \geqslant 0.5$ and $X_{1}^{(0)} / a \geqslant 10$, values which were too large for minuet to occur.

\subsection{Effect of capsule deformability}

Under given flow conditions, the capsule deformability is accounted for by the capillary number $\mathrm{Ca}$ and increases with it. Some global results are presented in the form of phase diagrams. For $X_{2}^{(0)}=0$ and $\left|X_{1}^{(0)}\right|=\left|X_{3}^{(0)}\right|$, the effect of varying the initial capsule separation and deformability is shown in figure $9(a)$. Minuet thus occurs approximately for $2<\left|X_{3}^{(0)}\right| / a<4$. For $\left|X_{3}^{(0)}\right| / a>4 \sim 5$, the capsule separation is so large that the relative velocity is very small and the capsule doublet configuration remains essentially stationary. The effect of $\mathrm{Ca}$ is complex: for a typical separation $\left|X_{3}^{(0)}\right| / a=2.6$ and up to $C a \leqslant 0.7$, a minuet takes place with one reversal for moderate values of deformability $(0.2<C a<0.7)$ or two (or more) reversals when $C a \leqslant 0.2$. This transition from one to two reversals when $\mathrm{Ca}$ is reduced has been illustrated in §3.2. However, for large capsule deformability $(\mathrm{Ca}>0.7)$, no motion reversal occurs: the capsules are so deformed and tilted towards the flow direction, that, when they overpass, their trajectory perturbation is small. The effect of $X_{2}^{(0)}$ is shown in figure $9(b)$ for the typical case $\left|X_{1}^{(0)}\right| / a=\left|X_{3}^{(0)}\right| / a=2.6$. Minuet occurs only for small values of $\left|X_{2}^{(0)}\right| / a$ and moderate $C a$, i.e. for small relative velocities between two capsules with moderate deformability. This limited range of minuet motion explains why it had not been detected before.

Note that the inherent deformability of a capsule, even when $\mathrm{Ca}$ is very small, makes it different from a rigid sphere. Consequently, it is impossible to find permanent 

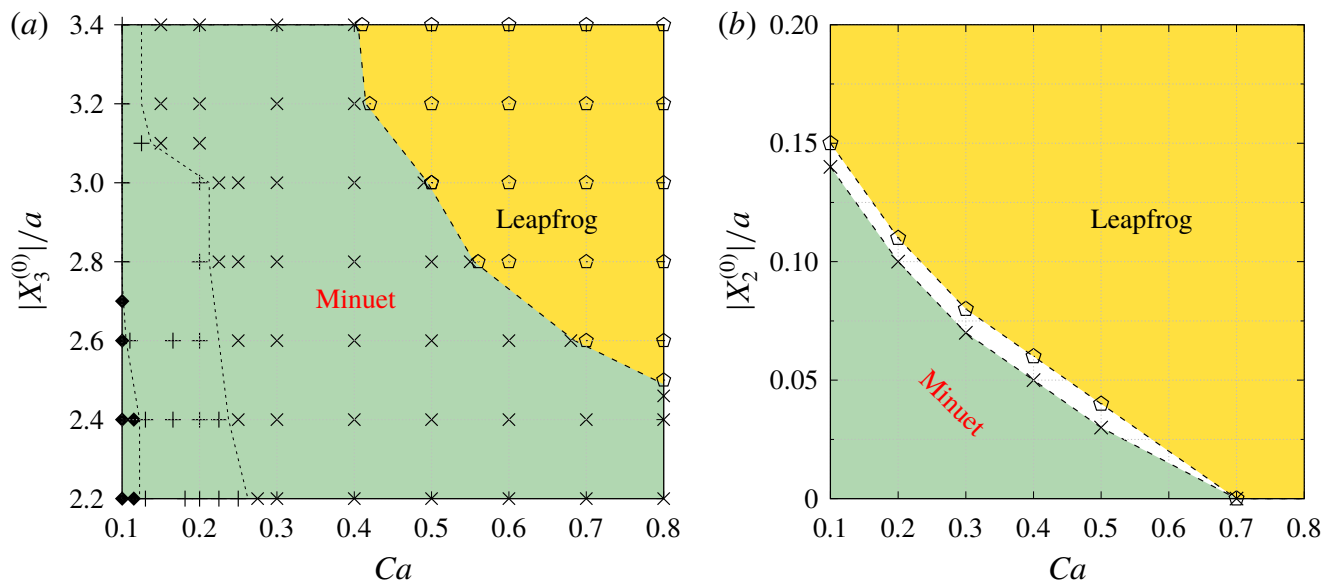

FIgURE 9. Phase diagrams for motion type: (a) motion type as a function of $C a$ and $X_{3}^{(0)}$ for $X_{1}^{(0)}=X_{3}^{(0)}$ and $X_{2}^{(0)}=0$. The points represent the positions of $G_{2}$ at rest: $\times$ one oscillation, + two oscillations, $\downarrow$ three oscillations. (b) Motion type as a function of $\mathrm{Ca}$ and $X_{2}^{(0)}$ for $\left|X_{1}^{(0)}\right| / a=\left|X_{3}^{(0)}\right| / a=2.6$.

capsule doublets like those predicted by Batchelor \& Green (1972b) for two spheres freely suspended in simple shear flow: indeed, such doublets can occur only for perfect spheres, as pointed out by the authors.

\subsection{Region of minuet motion}

The domain around $C_{1}$, where long term doublets are formed, is illustrated in figure 10 for $C a=0.3$. The sphere of radius $2.2 a$ centred on $G_{1}$ is an exclusion region, that leaves a space $0.2 a$ between the two capsules. When $X_{1}^{(0)}=X_{2}^{(0)}=0$, the capsule doublet separated by $X_{3}^{(0)}$, remains stationary. Whenever the centre of capsule $C_{2}$ is located in the yellow area, the two capsules will cross once (leapfrog motion) and separate. In the green area, the two capsules will remain close and oscillate a few times before eventually separating. For $\left|X_{3}^{(0)}\right| / a \gtrsim 4$, the interaction becomes weak, so that the doublet configuration remains essentially stationary. The minuet domain shown in figure 10 is in fact three-dimensional and extends in the $x_{2}$-direction over a small distance of order 0.06 a for $C a=0.3$ (see figure $9 b$ ). When $C a$ is decreased, the boundary between leapfrog and minuet motions does not change appreciatively, but the thickness of the three-dimensional minuet domain increases. Conversely, as shown in figure 10, when $\mathrm{Ca}$ increases, the boundary is tilted towards the $x_{3}$-axis and the minuet domain thickness decreases. Note that for $X_{2}^{(0)} \geqslant 0.5 a$, the whole region would be yellow (apart from the exclusion area).

An important consequence of the minuet motion is linked to the fact that it tends to push together two capsules that were initially distant and not expected to interact much. This point is illustrated in figure 6 where the initial separation $G_{1} G_{2}-2 a=1.7 a$ is decreased during crossing to a film with thickness approximately $0.2 a$, which is thin enough to lead to potential physico-chemical interactions or damage. 


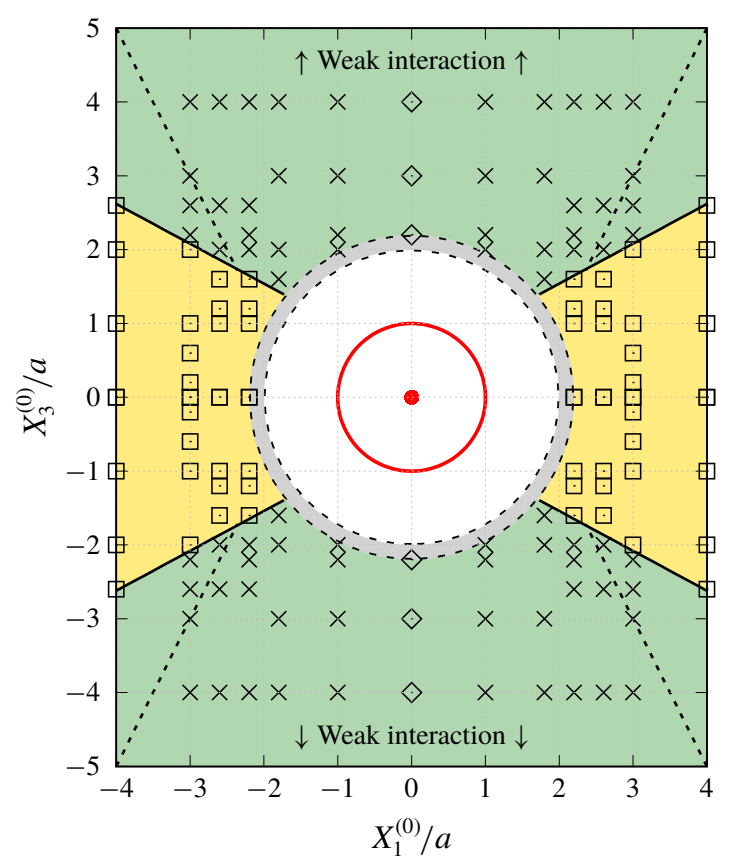

FIgURE 10. Domain of doublet formation around capsule $C_{1}$ for $X_{2}^{(0)}=0, C a=0.3$ and a $\mathrm{NH}$ membrane. The sphere of radius $2 a$ is a steric exclusion area, while the grey zone between the spheres of radii $2.2 a$ and $2 a$ is an exclusion region, that leaves a space $0.2 a$ between the two capsules. The points represent the positions of $G_{2}$ at rest, and the symbols indicate the motion type: $\times$ minuet, $\square$ leapfrog, $\diamond$ steady doublet. The position of the boundary between the two domains for $C a=0.5$ is indicated by a dash line.

\subsection{Effect of the membrane constitutive equation}

It is of interest to assess the influence of the wall constitutive law, as all the above results have been obtained for a neo-Hookean membrane. In particular, it is possible to assume that the principal tensions and elongations are related by the Skalak law (SK) (Skalak et al. 1973), which reads

$$
T_{1}=\frac{G_{s}}{\lambda_{1} \lambda_{2}}\left[\lambda_{1}^{2}\left(\lambda_{1}^{2}-1\right)+C \lambda_{1}^{2} \lambda_{2}^{2}\left(\lambda_{1}^{2} \lambda_{2}^{2}-1\right)\right]
$$

The surface shear elastic modulus is $G_{s}$ and the area dilation modulus is given by $K_{s}=(1+2 C) G_{s}$. The two laws (2.8) and (4.1) predict the same small deformation behaviour for $C=1$, but for large deformations, the $\mathrm{NH}$ law is strain softening, whereas SK law is strain hardening (Barthès-Biesel, Diaz \& Dhenin 2002). Correspondingly, for the same value of $\mathrm{Ca}$, the deformation of a single capsule in simple shear flow is larger for a $\mathrm{NH}$ membrane than for a SK one (Barthès-Biesel 2016).

We can then expect that the transition between a leapfrog and a minuet motion will happen for values of $\mathrm{Ca}$ that will be different for capsules with SK or NH membranes. In order to verify this prediction, we model the interaction of two capsules enclosed either by a SK membrane $(C=1)$ or a NH membrane, in the case $X_{1}^{(0)} / a=-3.0$, $X_{2}^{(0)}=0, X_{3}^{(0)} / a=-2.6$ and $C a=0.5$. As shown in figure $11(a)$, the NH capsules 

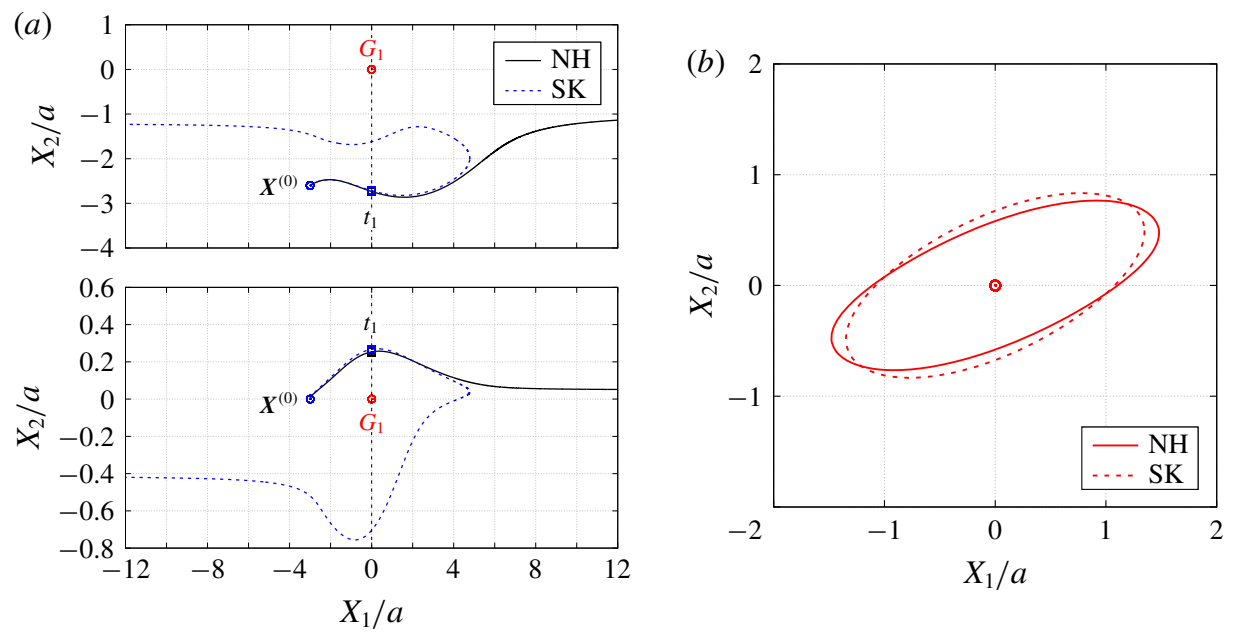

FIGURE 11. Interaction process of two capsules with a $\mathrm{NH}$ membrane or a SK membrane for $C a=0.5, X_{1}^{(0)} / a=-3, X_{2}^{(0)}=0, X_{3}^{(0)} / a=-2.6$. (a) The NH capsule does a sideways leapfrog motion, while the SK capsule does a motion reversal. $(b)$ Deformed profiles of the capsule $C_{1}$ at time $t_{1}$ defined by $X_{1}\left(t_{1}\right)=0$.

do a sideways leapfrog motion whereas the SK capsules undergo one oscillation and reverse their direction of motion. The explanation for this difference of behaviour is linked to the fact that the NH capsule is more deformed than the SK one (figure 11b), and that its trajectory displacement $\Delta_{2}$ is thus small enough to prevent it from going into the reverse flow region.

\section{Global effects: trajectory shift and doublet duration}

\subsection{Trajectory shift}

When two capsules with a NH membrane are in the same shear plane $\left(X_{3}^{(0)} / a=0\right)$, it is a well-established fact that, after crossing, the two capsules are irreversibly displaced from their initial trajectory: for a given value of $C a$, the trajectory shift $\delta_{2}=\left|X_{2}^{(f)}\right|-$ $\left|X_{2}^{(0)}\right|$ is maximum for $X_{2}^{(0)} / a=0$, decreases when $\left|X_{2}^{(0)}\right|$ increases and becomes almost zero for $\left|X_{2}^{(0)}\right| / a>2$ (Lac \& Barthès-Biesel 2008; Pranay et al. 2010; Omori et al. 2013; Gires et al. 2014). The effect of $X_{3}^{(0)} / a$ is mostly reported for the case $X_{2}^{(0)} / a=$ 0.5: then $\delta_{2}$ is maximum for $X_{3}^{(0)} / a=0$, decreasing to almost zero for $\mid X_{3}^{(0)} / a \geqslant 2$ (Lac \& Barthès-Biesel 2008; Gires et al. 2014). The trajectory shift along the vorticity direction $\delta_{3}=\left|X_{3}^{(f)}\right|-\left|X_{3}^{(0)}\right|$ is small and less than $0.1 a$ (see also figure 15). The effect of $C a$ is small and does not change the findings. The influence of the membrane constitutive law on the leapfrog motion in the shear plane was studied by Pranay et al. (2010), who compared the effect of a NH or SK law $(C=10)$ on the trajectory shift $\delta_{2}$. They found that, for the same $C a, \delta_{2}$ is larger for a SK law than for a $\mathrm{NH}$ one: this is due to the high apparent rigidity of the SK law, linked to a high value of the area dilation modulus.

The new results for $X_{2}^{(0)} / a=0$ are illustrated for $C a=0.3$ in figure 12 , where they are compared with the results of Lac \& Barthès-Biesel (2008), obtained for $X_{2}^{(0)} / a=$ 0.5 . For $X_{3}^{(0)} / a \leqslant 2$ when a leapfrog motion occurs, the shift $\delta_{2}$ decreases with $X_{3}^{(0)} / a$ 

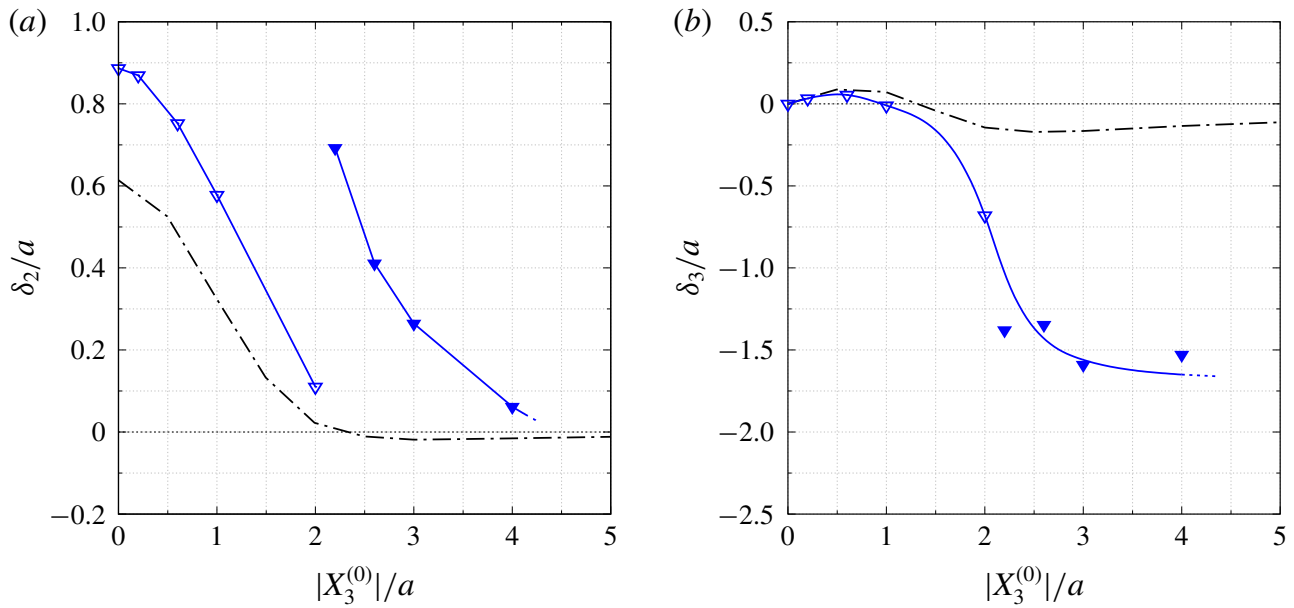

FIGURE 12. Irreversible trajectory shifts $\delta_{2}$ and $\delta_{3}$, computed at $\left|X_{1}^{(f)}\right| / a=10$, as a function of $X_{3}^{(0)}\left(X_{1}^{(0)} / a=-3.0, X_{2}^{(0)}=0, C a=0.3\right)$. Open symbols correspond to leapfrog motion, filled ones to minuet motion. The dash-dot line corresponds to the results of Lac $\&$ Barthès-Biesel (2008), obtained for $X_{1}^{(0)} / a=-10, X_{2}^{(0)} / a=0.5$ and $\beta=1.05$.

and is approximately $50 \%$ larger for $X_{2}^{(0)} / a=0$ than for $X_{2}^{(0)} / a=0.5$ (figure 12a): this is in agreement with the previously reported evolution of $\delta_{2}$ with $X_{2}^{(0)} / a$ and $X_{3}^{(0)} / a$. However, when the doublet motion evolves from leapfrog to minuet, a bifurcation takes place for $\left|X_{3}^{(0)}\right| / a=2.1 \pm 0.1: \delta_{2}$ jumps to values that are approximately one order of magnitude larger than the ones that would be expected from the previous $X_{2}^{(0)} / a=0.5$ results or from the prolongation of the leapfrog curve for $\delta_{2}$.

Similarly, whereas the shift $\delta_{3} / a$ remains small during the leapfrog motion, it becomes large and negative for $X_{2}^{(0)} / a \geqslant 2$ (figure $12 b$ ). This means that, at the end of the interaction process, the capsule $C_{2}$ is nearer the shear plane $x_{1} x_{2}$ than when the flow started. The minuet motion thus leads to a shift $\delta_{3}$ along the vorticity direction, which tends to reduce the initial distance between the two capsules, in opposition to a diffusive effect. But since the capsule separation in the $x_{3}$-direction is reduced, a sideways leapfrog motion takes place, with a resulting large value of $\delta_{2}$, and thus diffusive effects along the shear gradient direction. Decreasing $C a$ does not change much the above results. However, when the capsule deformability increases, the bifurcation occurs for increasingly large values of $\left|X_{3}^{(0)}\right| / a$ and is difficult to compute as the minuet becomes very slow (see $§ 5.2$ ).

The influence of the membrane law is shown in figure 13: the SK capsule undergoes a leapfrog motion for $\left|X_{3}^{(0)}\right| / a \leqslant 2$ and then makes a minuet for $\left|X_{3}^{(0)}\right| / a \geqslant 2.2$. The evolutions of $\delta_{2}$ are almost superimposed for a $\mathrm{NH}$ membrane $(\mathrm{Ca}=0.3)$ and for a SK membrane $(C a=0.5)$. When the SK capsule deformability increases to $C a=$ 1.0, the transition between leapfrog and minuet motions occurs further away, around $\left|X_{3}^{(0)}\right| / a \sim 3.5$. We can then conclude that, indeed, the effect of a strain hardening membrane would just be to shift the results to larger values of $C a$, without changing the essence of the interaction process. 


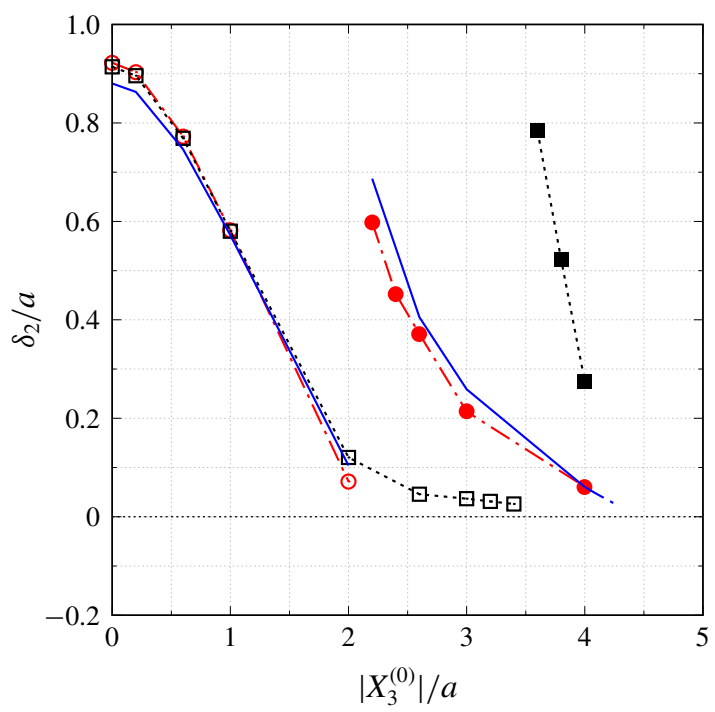

FIGURE 13. Trajectory shift $\delta_{2}$ as a function of $X_{3}^{(0)}$ for different membranes constitutive laws $\left(X_{1}^{(0)} / a=-3.0, X_{2}^{(0)}=0\right)$. The solid line corresponds to a neo-Hookean membrane with $C a=0.3$ (figure 12). The dashed lines correspond to a Skalak membrane $(C=1)$; $\bigcirc C a=0.5, \square C a=1.0$. Open symbols: leapfrog motion; filled symbols: minuet motion.

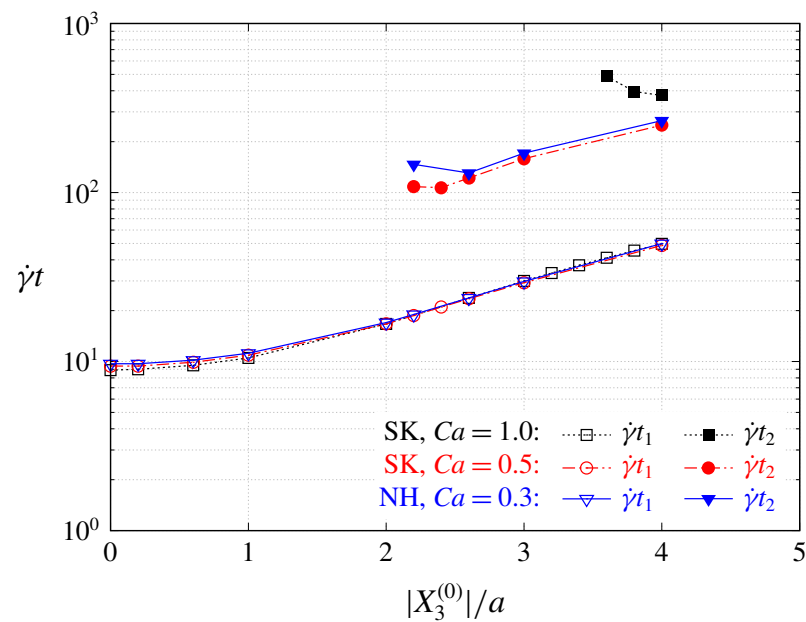

FIgURE 14. Effect of $\mathrm{Ca}, X_{3}^{(0)}$ and constitutive law on the times $\dot{\gamma} t_{1}$ and $\dot{\gamma} t_{2}$ of first and second crossings $\left(X_{1}^{(0)} / a=-3.0\right.$ and $\left.X_{2}^{(0)} / a=0\right)$.

\subsection{Doublet duration}

The minuet motion, when it occurs, is very slow, as shown in figure 14 for $X_{1}^{(0)} / a=$ -3.0 and $X_{2}^{(0)} / a=0$. The time $\dot{\gamma} t_{1}$, at which the first crossing occurs, increases with the separation $\left|X_{3}^{(0)}\right| / a$, but does not depend on $C a$, the membrane law or the type of motion (leapfrog or minuet). The fact that $\dot{\gamma} t_{1}$ increases with $\left|X_{3}^{(0)}\right| / a$ is due to 

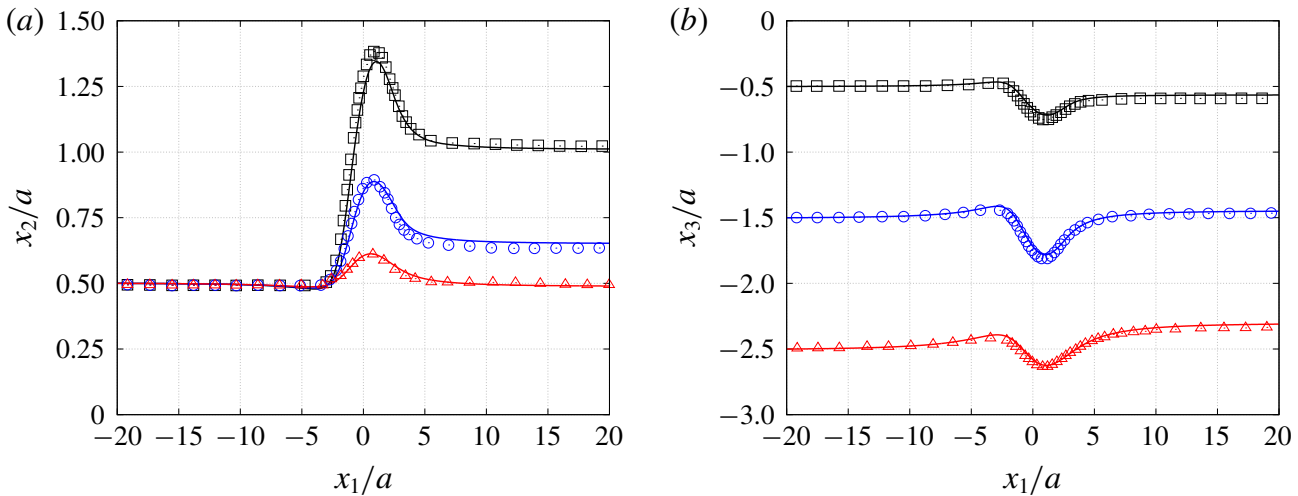

FIGURE 15. Projection of $G_{2}$ trajectories for $\beta=1.05, C a=0.45$ and different initial positions. Capsule $C_{2}$ starts from $X_{1}^{(0)} / a=-20, X_{2}^{(0)} / a=0.5$ and different $X_{3}^{(0)}$. The comparison between the results of Lac \& Barthès-Biesel (symbols) and the present results (lines) shows very good agreement.

the fact that the relative velocity of $C_{2}$, which is initially zero, builds up from a flow perturbation (due to $C_{1}$ ), the intensity of which decreases with the square of the distance between the two capsules. The time $\dot{\gamma} t_{2}$ of the second crossing is quite large and increases significantly with capsule deformability and distance.

This means that the two capsules remain close to each other for a long time, while drifting slowly. When $\left|X_{3}^{(0)}\right| / a \geqslant 4, \dot{\gamma} t_{2}$ becomes too large to be reliably computed (the values of $\dot{\gamma} t_{2}$ for $C a=1.0$ and a SK membrane are very high, and are included here to illustrate the phenomenon).

\section{Discussion and conclusion}

The situation that we have studied pertains to a semi-dilute suspension of capsules that is suddenly put in motion by a simple shear flow. The novel aspect of our work is that we consider a pair of nearby capsules with their centres in (or near) the plane normal to the velocity gradient $\left(X_{2}^{(0)} \sim 0\right)$. This capsule configuration had never been studied before, because it entails long computations. Indeed, the only results on capsule interaction off the shear plane had been obtained for capsules with a significant relative velocity, which prevented minuet from occurring and which led to weak capsule interactions and small trajectory displacement.

When $X_{2}^{(0)} \sim 0$, the important results are:

(i) The capsule pair can remain stable for a long time, while dancing a minuet.

(ii) When they separate, the capsules can reverse direction.

(iii) When one or more oscillations occur, the irreversible trajectory shift is large.

(iv) This minuet dance progressively leads the capsules to closely interact and deform significantly.

(v) The less deformable the capsule, the more prone it is to do a minuet.

(vi) The zone where a minuet can occur has been identified.

During the close interaction, the film thickness between the two capsules is of the order of $\sim 0.1 a-0.2 a$. For small capsules, this may lead to physico-chemical interaction. 
In order to make the problem consistent, we considered as an initial condition a suspension of spherical capsules at rest, and suddenly started the flow. When the capsules are pre-deformed to the profile that they would have if they were alone in the flow, we have verified that they take the same motion (leapfrog or minuet) as if they were initially spherical: the only difference is that, for pre-deformed capsules, the first close interaction happens $\dot{\gamma} t \leqslant 5$ earlier than for spherical capsules. It follows that the minuet interaction is not restricted to the transient start of the flow of a suspension. For example, from figure 6, we note that, at the end of the interaction, when $C_{2}$ is located at $X_{1} / a=5.0, X_{2} / a=0.54, X_{3} / a=-1.1$, it has almost recovered its equilibrium shape. Suppose that $C_{2}$ then meets a new capsule $C_{3}$, located in the vicinity of $X_{1} / a=7.6, X_{2} / a=0.54, X_{3} / a=1.5$. Being in the same relative configuration as $C_{1}$ and $C_{2}$ were at time $t=0$, capsules $C_{2}$ and $C_{3}$ will do a minuet. We can thus expect minuet motions in semi-dilute suspensions.

In conclusion, we have shown a novel and unexpected effect in the pair interaction of two capsules, that depends on the relative position of the two particles. It would be interesting to check experimentally the existence of long lasting capsule doublets that do a minuet motion.

\section{Acknowledgements}

This work was supported by the European Research Council (ERC) Consolidator grant (MultiphysMicroCaps, no. 772191), by the National Natural Science Foundation of China (no. 11402084) and by the China Scholarship Council (Visiting Scholar Scholarship of X.-Q.H.).

\section{Declaration of interests}

The authors report no conflict of interest.

\section{Supplementary movies}

Supplementary movies are available at https://doi.org/10.1017/jfm.2020.181.

\section{Appendix}

The results of the BI-FE model (1280 elements) are first compared to those obtained by Lac et al. (2007) and Lac \& Barthès-Biesel (2008) for two pre-inflated capsules with a $\mathrm{NH}$ membrane and radius $\beta a$, where $\beta$ is the inflation ratio. The pre-stress is created by means of an internal pressure $p_{0}$, which leads to an isotropic elastic tension $T_{0}=p_{0} a / 2$, given by Laplace's law. For a neo-Hookean membrane, $T_{0}=6 G_{s}(\beta-1)$ in the limit of small inflation. The trajectories of $G_{2}$ obtained with the two methods show very good agreement, as illustrated in figure 15 for $C a=0.45$.

As the minuet motion is a novel phenomenon, which occurs over fairly long times, it is of importance to verify that the trajectories do not suffer from error accumulation over time. The test case consists of two capsules enclosed by a SK membrane $(C=1)$ with $C a=0.5, X_{1}^{(0)} / a=-3.0, X_{2}^{(0)}=0$ and $X_{3}^{(0)} / a=-2.4$. As shown in figure 13, those capsules undergo a minuet with one reversal. The choice of a SK law rather than a NH one enables us to use larger values of $\mathrm{Ca}$, and thus larger time steps for similar trajectories. As shown in figure $16(a)$ for 1280 elements on the capsule surfaces, decreasing the time step $\dot{\gamma} \Delta t$ from $2 \times 10^{-3}$ to $5 \times 10^{-4}$ has no effect on the trajectory. Conversely, we keep the same time step $\dot{\gamma} \Delta t=2 \times 10^{-3}$ 

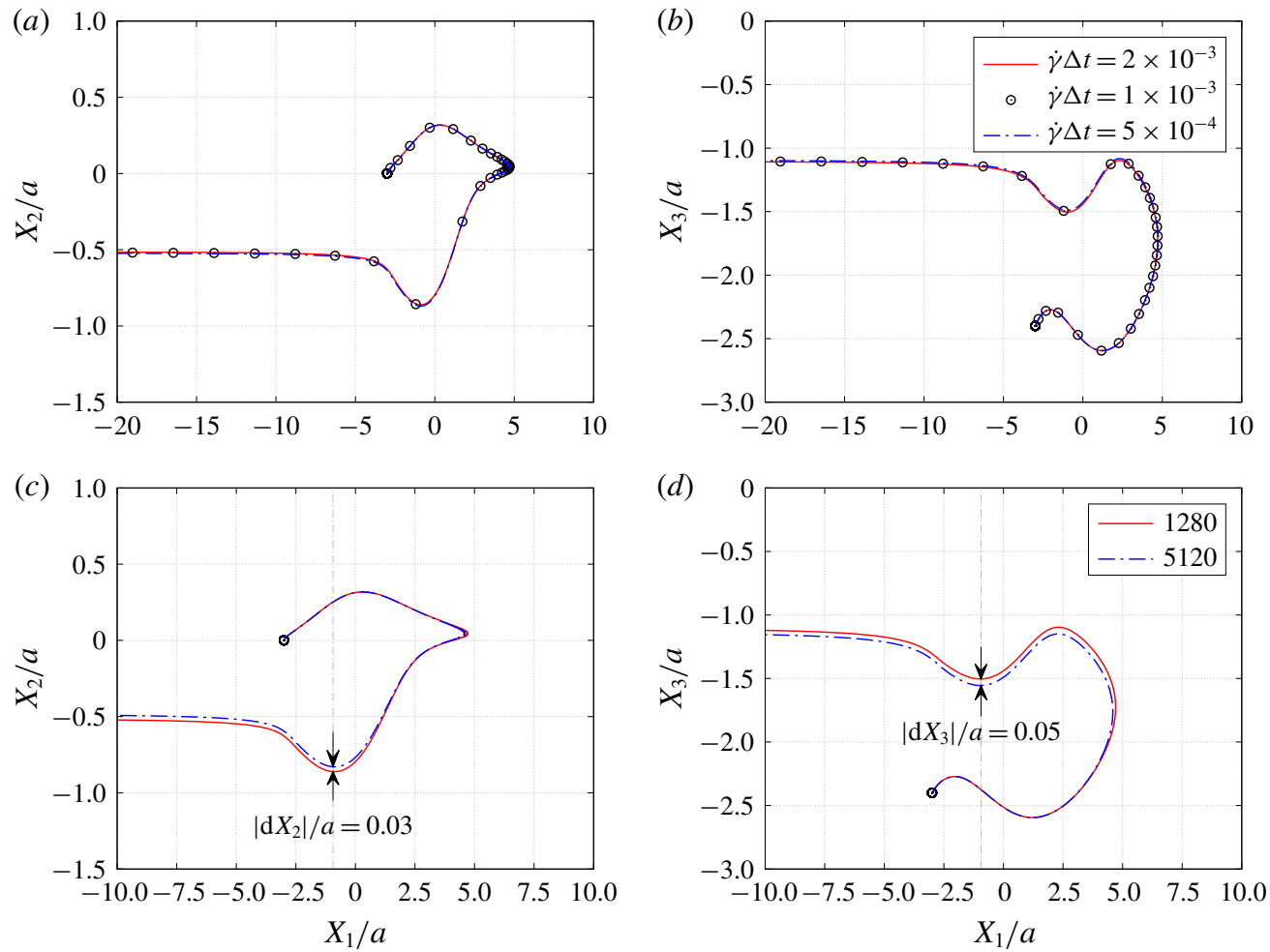

FIGURE 16. Effect of numerical parameters on the $G_{2}$ trajectory, projected on the $x_{1} x_{2}$ and $x_{1} x_{3}$-planes for SK membranes, for $C a=0.5, X_{1}^{(0)} / a=-3.0, X_{2}^{(0)} / a=0, X_{3}^{(0)} / a=-2.4$. $(a, b)$ Effect of time step for 1280 elements. $(c, d)$ Effect of mesh refinement for $\dot{\gamma} \Delta t=$ $2 \times 10^{-3}$.

and compare the trajectories obtained with two spatial meshes with 1280 or 5120 elements, corresponding to mesh sizes $\Delta h_{c}=O(0.1 a)$ and $O(0.05 a)$, respectively. As shown in figure $16(b)$, the trajectories differ by at most one (fine) mesh size at the interaction point. We conclude that the trajectories that are presented in this study using 1280 elements and $\dot{\gamma} \Delta t=5 \times 10^{-4}$, are reliable and that the final trajectory shifts $\delta_{2} / a$ and $\delta_{3} / a$ have an error of the order of $0.05 a$.

\section{REFERENCES}

BARTHÈs-BIESEL, D. 2016 Motion and deformation of elastic capsules and vesicles in flow. Annu. Rev. Fluid Mech. 48 (1), 25-52.

BARThÈs-BIESEl, D., DiAZ, A. \& DHEnin, E. 2002 Effect of constitutive laws for two dimensional membranes on flow-induced capsule deformation. J. Fluid Mech. 460, 211-222.

BATChELOR, G. K. \& GREen, J. T. $1972 a$ The determination of the bulk stress in a suspension of spherical particles to order $c^{2}$. J. Fluid Mech. 56 (2), 401-427.

BATChELOR, G. K. \& GREen, J. T. $1972 b$ The hydrodynamic interaction of two small freely-moving spheres in a linear flow field. J. Fluid Mech. 56 (2), 375-400.

Doddi, S. K. \& BAGCHI, P. 2008 Effect of inertia on the hydrodynamic interaction between two liquid capsules in simple shear flow. Intl J. Multiphase Flow 34 (4), 375-392. 
Drescher, K., Leptos, K. C., Tuval, I., Ishikawa, T., Pedley, T. J. \& Goldstein, R. E. 2009 Dancing Volvox: hydrodynamic bound states of swimming algae. Phys. Rev. Lett. 102, 168101.

Dupont, C., Delahaye, F., Barthès-Biesel, D. \& Salsac, A.-V. 2016 Stable equilibrium configurations of an oblate capsule in simple shear flow. J. Fluid Mech. 791, 738-757.

Dupont, C., SAlsac, A.-V. \& BARThÈs-BIESEl, D. 2013 Off-plane motion of a prolate capsule in shear flow. J. Fluid Mech. 721, 180-198.

Gires, P. Y., Srivastav, A., Misbah, C., Podgorski, T. \& Coupier, G. 2014 Pairwise hydrodynamic interactions and diffusion in a vesicle suspension. Phys. Fluids 26 (1), 013304.

Guazzelli, E. \& Morris, J. F. 2012 A Physical Introduction to Suspension Dynamics. Cambridge University Press.

Guido, S. \& Simeone, M. 1998 Binary collision of drops in simple shear flow by computer-assisted video optical microscopy. J. Fluid Mech. 357, 1-20.

Hu, X.-Q., SAlsac, A.-V. \& BARThÈs-BIESEL, D. 2012 Flow of a spherical capsule in a pore with circular or square cross-section. J. Fluid Mech. 705, 176-194.

Kantsler, V., Segre, E. \& Steinberg, V. 2008 Dynamics of interacting vesicles and rheology of vesicle suspension in shear flow. Europhys. Lett. 82 (1), 58005.

LAC, E. \& BARTHÈS-BIESEL, D. 2008 Pairwise interaction of capsules in simple shear flow: threedimensional effects. Phys. Fluids 20 (4), 040801.

Lac, E., Morel, A. \& BARThÈs-Biesel, D. 2007 Hydrodynamic interaction between two identical capsules in simple shear flow. J. Fluid Mech. 573, 149-169.

Loewenberg, M. \& Hinch, E. J. 1997 Collision of two deformable drops in shear flow. J. Fluid Mech. 338, 299-315.

Olapade, P. O., Singh, R. K. \& SARKar, K. 2009 Pairwise interactions between deformable drops in free shear at finite inertia. Phys. Fluids 21 (6), 063302.

Omori, T., Ishikawa, T., Imai, Y. \& Yamaguchi, T. 2013 Shear-induced diffusion of red blood cells in a semi-dilute suspension. J. Fluid Mech. 724, 154-174.

Pranay, P., Anekal, S. G., Hernandez-Ortiz, J. P. \& Graham, M. D. 2010 Pair collisions of fluid-filled elastic capsules in shear flow: effects of membrane properties and polymer additives. Phys. Fluids 22 (12), 123103.

SingH, R. K. \& SARKAR, K. 2015 Hydrodynamic interactions between pairs of capsules and drops in a simple shear: effects of viscosity ratio and heterogeneous collision. Phys. Rev. E 92 (6), 063029.

Skalak, R., Tozeren, A., Zarda, R. P. \& Chien, S. 1973 Strain energy function of red blood cell membranes. Biophys. J. 13, 245-264.

WAlter, J., SAlsaC, A.-V. \& BARTHÈs-Biesel, D. 2011 Ellipsoidal capsules in simple shear flow: prolate versus oblate initial shapes. J. Fluid Mech. 676, 318-347.

Walter, J., Salsac, A.-V., Barthès-Biesel, D. \& Le Tallec, P. 2010 Coupling of finite element and boundary integral methods for a capsule in a Stokes flow. Intl J. Numer. Meth. Engng 83 (7), 829-850. 